\title{
BiSON: A Bioinspired Self-Organizing Network for Dynamic Auto-Configuration in 5G Wireless
}

\author{
Abhishek Roy $\mathbb{D}^{\mathbb{D}},{ }^{1}$ Navrati Saxena ${ }^{(D)},{ }^{2}$ Bharat J. R. Sahu $\left(\mathbb{D},{ }^{3}\right.$ and Sukhdeep Singh ${ }^{4}{ }^{4}$ \\ ${ }^{1}$ MediaTek USA Inc., San Jose, USA \\ ${ }^{2}$ Sungkyunkwan University, Suwon, Republic of Korea \\ ${ }^{3}$ Kyungpook National University, Republic of Korea \\ ${ }^{4}$ Samsung R\&D Institute India-Bangalore (SRI-B), Bangalore, India
}

Correspondence should be addressed to Navrati Saxena; navrati@skku.edu

Received 17 June 2018; Accepted 17 September 2018; Published 7 November 2018

Academic Editor: Enrico M. Vitucci

Copyright (c) 2018 Abhishek Roy et al. This is an open access article distributed under the Creative Commons Attribution License, which permits unrestricted use, distribution, and reproduction in any medium, provided the original work is properly cited.

\begin{abstract}
Emerging 5G wireless networks are expected to herald significant transformation in industrial applications, with improved coverage, high data rates, and massive device capacity. However, the introduction of 5G wireless makes the network configuration, management, and planning extremely challenging. For efficient network configuration, every cell needs to be allocated a particular Physical Cell Identifier (PCID), which is unique in its vicinity. Wireless standards (e.g., 3GPP) typically specify a limited number of PCIDs. However, the number of cells in 5G Ultradense Networks (UDN) is expected to significantly outnumber these limited PCIDs. Hence, these PCIDs need to be efficiently allocated among the myriad of cells, such that two cells which are neighbors or neighbor's neighbor are assigned with different PCIDs. This complicated network configuration problem becomes even more complex by dynamic introduction and removal of $5 \mathrm{G}$ small cells (e.g., micro, femto, and pico). In this paper, we introduce BiSON, a new Bioinspired Self-Organizing Solution for automated and efficient PCID configuration in 5G UDN. Using two different extensions, namely, "always near-optimal" and "heuristic," we explain near-optimal and dynamic auto-configuration in computationally feasible time, with negligible overhead. Our extensive network simulation experiments, based on actual 5G wireless trials, demonstrate that the proposed algorithm achieves better optimality (minimum PCIDs in use) than earlier works in a reasonable computational complexity.
\end{abstract}

\section{Introduction}

Next generation 5G wireless [1] envisions revolutionizing industrial applications, like robotics and smart grids, by providing manifold improvement in latency, data rates, and device capacity. While existing wireless networks are mostly designed for human-centric communications, emerging $5 \mathrm{G}$ wireless aims to provide default support to Machine-toMachine (M2M) communications and Internet-of-Things (IoT). Network densification [1] is already identified as a key factor in meeting explosive device growth, with high spectral efficiency. However, commercial roll out of $5 \mathrm{G}$ Ultradense Networks (UDN), consisting of a wide number of relatively small cells, makes the network configuration and management extremely complex. Moreover the dynamic introduction and removal of small 5G cells make it almost impossible to manually configure and manage the already complex networks. Thus, automated configuration and management are considered as two integral features of emerging 5G wireless systems. Self-Organizing Networks (SON) [2] are gradually becoming popular by offering selfconfiguration, self-optimization, and self-healing of cellular networks, thereby reducing both capital expenditure (capex) and operating expenditure (opex) in network planning, deployment, and operation.

Figure 1 demonstrates a 5G UDN, connected with SON server for self-configuration and management. Physical Cell Identifier (PCID) is one of the most important cell configuration parameters. Without an assigned PCID, the mobile device cannot even detect the cell, thus failing to setup any radio communication. The number of available PCIDs is generally limited. While the $5 \mathrm{G}$ standards are yet to be 


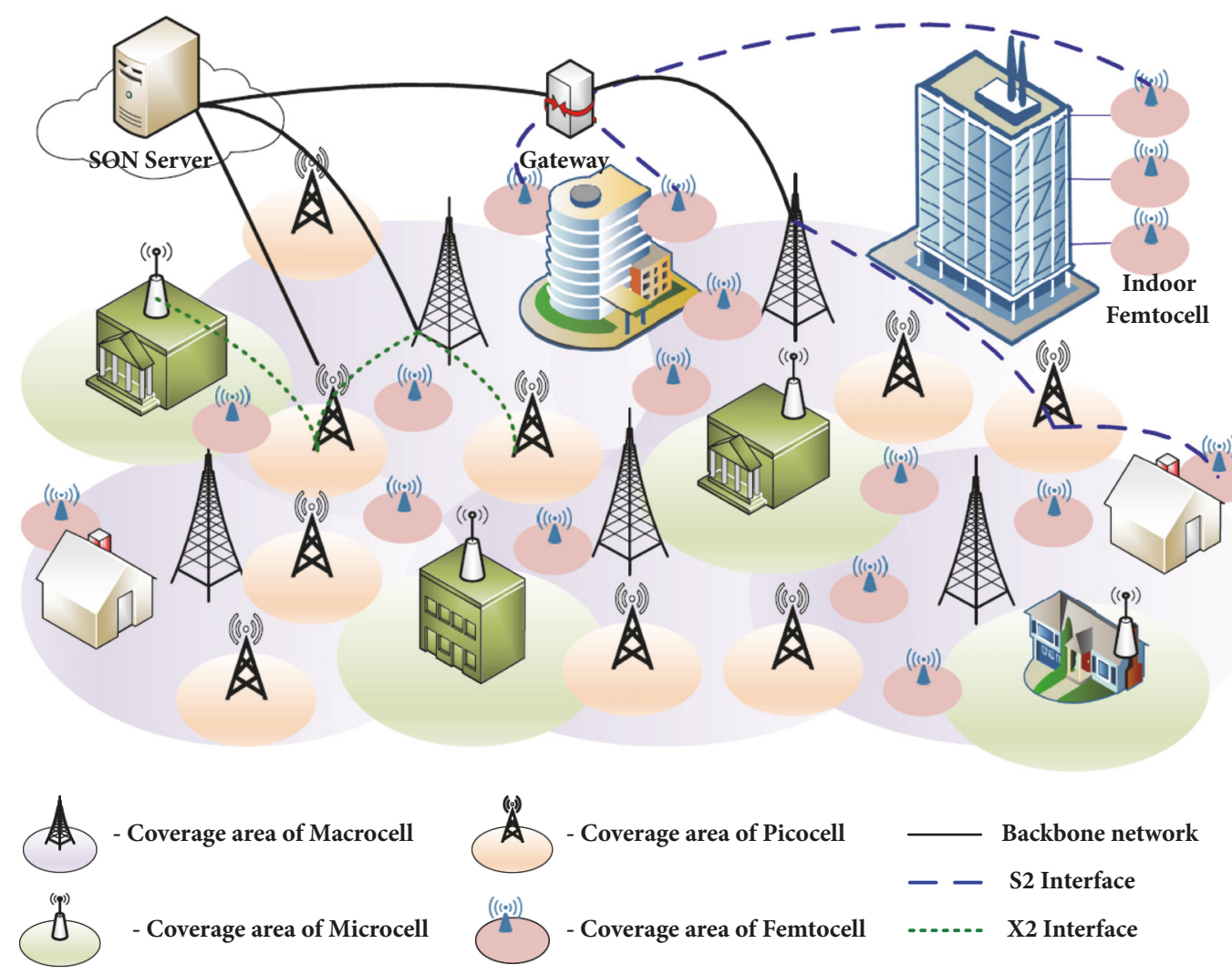

FIGURE 1: 5G ultradense network with SON.

defined, current 3GPP specification (Self-configuration and self-optimizing network use cases and solutions. 3GPP TS $36.902,2008$.) allows only 504 PCIDs for network configuration. This raises a significant new challenge in emerging $5 \mathrm{G}$ wireless configuration, as the number of cells in $5 \mathrm{G}$ UDN is expected to be more than the available PCIDs by several multitudes. Thus, an efficient, dynamic, and optimal PCID configuration among the myriad of $5 \mathrm{G}$ cells is of utmost important. Collision-free and confusion-free (Selfconfiguration and self-optimizing network use cases and solutions. 3GPP TS 36.902, 2008.) are two necessary constraints associated with PCID allocation [3].

(1) The connotation of collision-free is "two neighboring cells should not have the same PCID". An illustration of intricacy arising from colliding PCID is illustrated here: received signals from available base stations (BSs) are monitored by mobile user equipment (UE). Subsequently, handover decisions between two BSs, and potential handover candidate BS selections are performed based on this signal measurement. In the case of identical PCIDs, the cells with weaker signals are discarded by the UE, thus a potential handover candidate recognition fails. Ultimately, when the signal of the potential cell gets stronger than the current cell, the UE tries to use the stronger signal, and the connection fails (EUTRAN Overall Description. 3GPP TS 36.300. 2008).
(2) The confusion-free,, on the other hand, mandates that a cell should not have two neighbors configured with the same PCID. Every BS maintains an automated neighbor relation (ANR) table, containing the information of neighboring cells for possible handover. This includes PCID of potential handover candidates. For a BS with two neighboring cells having identical PCID, the BS keeps only one of those cells in the ANR table. Hence, handover to the cell whose PCID is not in the ANR table fails.

A massive number of heterogenous cells in $5 \mathrm{G}$ are bound to increase the complexity of the already complicated problem. A survey of the recent research works in self-organization and management [4] reveals that a large number of academic researches and international projects reflect the importance of PCID allocation with self-configuration, plug-and-play, self-optimization, self-healing (in case of failure), and automated network management. This motivates us to look into the dynamic self-configuration problem in next generation of 5G wireless networks. In particular, we take a step forward to answer the following fundamental questions. (a) How can we define optimal self-configuration problem in 5G UDN? (b) What is the complexity of optimal self-configuration? (c) How to design feasible, near-optimal solutions for selfconfiguration in 5G UDN. (d) Is the efficiency vs. overhead trade-off in the solutions enough for real implementation? In this paper we will discuss our answers to these fundamental questions. More specifically our contributions are as follows: 
TABLE 1: Major works.

\begin{tabular}{ll}
\hline \multicolumn{1}{c}{ SON: key challenges and solution } \\
\hline Network management & RRA, HO, interference, antenna downtilt, \& load management [2] \\
\hline Graph coloring & RRA \& antenna downtilt [6] \\
\hline Game theory & Square graph coloring for PCI [3, 4] \\
\hline Location based PCI & Game theoretic minimum collisions assignment [7] \\
\hline KPI Selection & Geo-location based PCI conflict resolution [8, 9] \\
\hline Fault diagnosis (self-healing) & Method to automatically select KPIs for self-healing functions [10] \\
\hline Survey & Ensemble learning based system [11] \\
\hline & Machine learning algorithms applied to SON in last 15 years [12] \\
\hline Survey & Bio-inspired solution: applications in wireless network \\
\hline Wireless network & Networking solutions [13, 14], vehicular network routing [15] \\
\hline WSN & Resource scheduling [16, 17], cell planning [18], load balance [19] \\
\hline
\end{tabular}

(1) We first discuss and formulate the optimal PCID configuration problem in 5G UDN. Subsequently, we prove that the global optimal solution is computationally complex and NP-complete.

(2) We design a new customized genetic algorithm (GA) to model the optimal PCID configuration problem. Our strategy iteratively reduces the number of PCID using a set of new operations called Fusion Operation. We use the concept of virtual cell or virtual cell cluster [5] to resolve the possible future collisions and confusions, arising from dynamic, new small cell installations (e.g., plug-and-play of 5G indoor cells).

(3) Next, we propose biologically inspired SON (BiSON), a genetic algorithm (GA) based solution which iteratively explores the search space and improves the quality of the candidate solutions in every iteration. BiSON algorithm achieves a near-optimal solution in computationally feasible time.

(4) We perform detailed stochastic modeling and analysis of BiSON to point out the associated convergence speed.

(5) Rigorous simulation of 5G UDN using 5G channel models and RF parameters derived from actual field trials demonstrate the efficiency of our solution. The results dynamically show $53 \% \sim 55 \%$ improvement in auto-configuration more than previous works $[3,5]$ in computationally feasible time.

The remainder of the paper is organized as follows. Section 2 reviews the major existing works in automated self-configuration of cellular networks. Dynamic, optimal PCID allocation problem and its complexity are illustrated in Section 3. Section 4 details our proposed GA modeling schemes involving a new chromosome design and fitness evaluation. We describe our proposed algorithm which efficiently uses GA to obtain the near-optimal solution in Section 5. Subsequently, we also analyze the convergence and speed of our proposed algorithm in Section 6. Simulation results in Section 7 substantiate the dynamism and efficiency of our scheme. Finally, Section 8 concludes the paper.

\section{Existing Works on Self-Configuration in Cellular Wireless}

Table 1 shows the summary of major existing works on SON and bioinspired approaches in wireless networks. Geolocation based allocation $[8,9]$, game-theory based minimum collision assignment [7], and graph coloring optimization $[3,4]$ are some of the PCID conflict resolution approaches available in literature. SON is gradually coming up as the panacea for the complex configuration and management challenges of 5G UDN [27]. LTE is the first technology to use the SON features, with inherent support of ANR detection. A recent review on SON [2] demonstrates the wide number of industrial and academic research activities on autonomic network configuration and self-management. SON is also providing promises for autonomous capacity enhancement, handover (HO) optimization, interference control, antenna downtilt, and radio resource allocation (RRA) [2, 6]. Similarly, SOCRATES (Socrates-FP7, http://www.fp7socrates.eu, (October, 2018).) and E32 are major joint initiatives for the design and development of self-configurations, optimization, and healing of wireless networks. Research work of [10] provides the technique for selecting the Key Performance Indicators (KPIs) used as SON function inputs under various network conditions. Authors in [11] focused on self-healing characteristic of SON. They targeted fault diagnosis function of self-healing and proposed an ensemble learning based system for the same. Another recent survey [12] focuses on machine learning algorithms applied to SON in terms of learning solutions and use cases. It also compares various machine learning algorithms on the basis of different SON metrics.

Bioinspired solutions are a class of algorithms imitating some biological mechanisms to solve complex optimization problems. An extensive survey of bioinspired solution for the communications network is presented in $[13,15]$. Bioinspired solutions have been used in wireless networks for scheduling and resource allocation [16, 17], optimal cell planning [18], BS deployment [18], and load balancing [19]. Similarly, ant-colony based optimization [16], bee-colony based optimization [19], and genetic algorithms [28] are the major 
bioinspired tools used for learning troubleshooting fuzzy rules and optimization in self-healing wireless networks. On the other hand, wireless sensors networks use bioinspired solution for optimal clustering [21], routing path selection, and energy efficient coverage scheduling [20]. Reference [14] provides an overview of researchers working on solutions related to bioinspired networking along with practical relevance and capabilities of bioinspired networking. Authors in [22] present an idea of utilizing selfsynchronization techniques inculcated from biological systems for optimal wireless sensor network decisions. Bioinspired trust and reputation model for WSN, i.e., BTRMWSN [23], is an ant-colony system based model providing reputation and trust in WSNs to assure security in WSNs.

A close look into most of the existing literature of cell configurations reveals that most of the existing solutions perform some local adjustments, involving PCID updates in only one of the conflicting cells. As the algorithms are not designed for minimizing collision and confusion, the resulting solutions do not guarantee any avoidance of consecutive PCID conflicts after the commercial deployment, i.e., during the network's operational phase. These solutions often do not even guarantee if a valid configuration could be at all found or if the changes will result in oscillating reconfigurations through large parts of 5G UDN. Moreover, the manifold increase in Quality of Experience (QoE) requirements and the increased network complexity require more efficient and low overhead PCID distribution in 5G UDN. This motivates us to look for an efficient, bioinspired solution for the complex PCID allocation problem.

\section{Complexity of Optimal PCID Allocation}

We consider a dense $5 \mathrm{G}$ wireless network, with macrocells, small cells, and the SON server. For formulating the optimal PCID assignment problem, we assume $\Upsilon$ cells and $\wp$ available PCIDs. As $\Upsilon>\wp$, more than one cell need to have the same PCID. However, assignment of the same PCID to any two neighboring cells creates a collision in frequency. Similarly, the assignment of the same PCID to multiple cells which are neighbor's neighbor leads to a confusion in managing measurement report during handover. Based on this discussion, we can now define the optimal PCID allocation problem in 5G UDN as follows.

Given a $5 \mathrm{G}$ network topology with a set of $\Upsilon$ cells and $\wp$ PCIDs, the optimal PCID allocation problem is to allocate the minimum number of PCIDs into the set of all cells, so that the entire network is (i) collision-free and (ii) confusionfree.

Note that the collision-free and confusion-free constraints need to be maintained not only during the initial assignment, but also during dynamic introduction (plugand-play) and removal of new small cells, without compromising the network performance. Unfortunately, this dynamic PCID allocation problem turns out to be NP-hard [29]. We prove this by mapping "vertex $k$-coloring Problem" [30] - a well-known NP-complete problem [29] to our PCID
TABLE 2: Mapping between PCID allocation and Vertex k-coloring.

\begin{tabular}{lc}
\hline Vertex k-coloring & Optimal PCID allocation \\
\hline Set of $k$ color & Set of $\wp$ PCID \\
\hline Set of vertices $\mathscr{V}$ & Set of $\Upsilon$ cells \\
\hline Set of $\mathscr{E}$ edges & Collision and confusion free \\
\hline Graph $G$ & 5G UDN topology \\
\hline
\end{tabular}

allocation problem. The vertex coloring problem can be defined as follows: For a graph $G(\mathscr{V}, \mathscr{E})$, with $\mathscr{V}$ vertices and $\mathscr{E}$ edges, vertex $k$-coloring problem is an assignment of one of the $k$ available colors to each vertex in $\mathscr{V}$, in such a way that no edge connects two identically colored vertices.

Now we map the vertex $k$-coloring to our optimal PCID allocation problem as follows.

(i) Map the set of $k$ colors to $\wp$ PCID, i.e., $k \longmapsto \wp$.

(ii) Map the set of $\mathscr{V}$ vertices in the graph to set of $\Upsilon$ cells, i.e., $\mathscr{V} \longmapsto \Upsilon$.

(iii) Map the set of $\mathscr{E}$ edges to the combination of both "collision-free" and "confusion-free" property, i.e., $\mathscr{E} \longmapsto$ collision-free or confusion-free.

(iv) Map the graph $G$ to the entire $5 \mathrm{G} U \mathrm{UDN}$, i.e., $\mathscr{G} \longmapsto 5 G$ UDN.

The complexity of the problem is similar to the problem of finding number of w-length words, over an alphabet of $k$ letters where each letter appears in each word at least once. As mentioned in [31], the complexity and search space are bounded by

$$
\prod_{w, k}=\sum_{i=0}^{k}(-1)^{k}\left(\begin{array}{l}
k \\
i
\end{array}\right)(k-i)^{w}
$$

Table 2 depicts mapping between PCID allocation and vertex $\mathrm{k}$-coloring. The goal of the solution is to find the lowest $k$ that results in a valid coloring. This $k$ is often called the chromatic number [30]. Thus, we can now say that the dynamic PCID allocation problem in next generation $5 G$ wireless $U D N$ is NPhard with exponential complexity. The exponential complexity of (1) compels us to look for a near-optimal solution in computationally feasible time. In the following section, we introduce our new GA-based solution to capture, model, and analyze the self-configuration of $5 \mathrm{G}$ cells.

\section{Modeling with Genetic Algorithm}

In this section, we introduce the general idea of GA. Subsequently, we model our PCID allocation problem into customized GA concept.

4.1. Genetic Algorithms. Bioinspired algorithms [32] are gradually emerging as one of the most powerful methods to solve many real world optimization problems. It mimics our mother nature to solve the practical problems. EvolutionaryGA [32] is such a bioinspired approach, which mimics the Darwinian evolutionary principles, like Survival of the fittest 

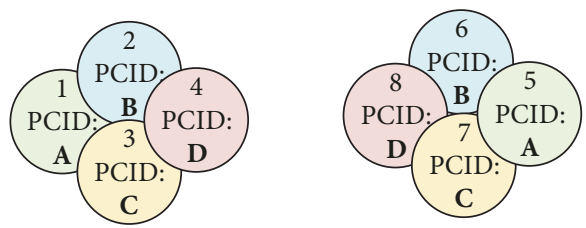

(a)

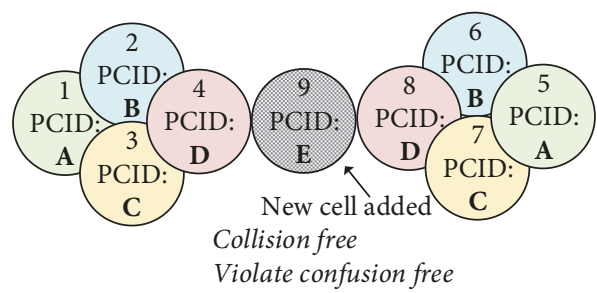

(b)

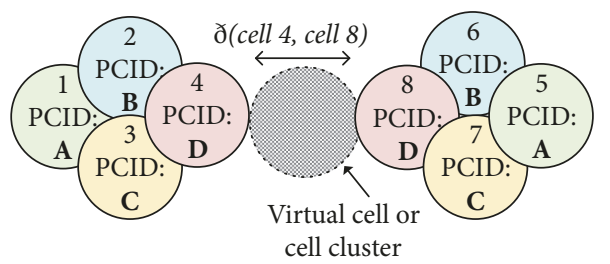

(c)

Figure 2: (a) Example cells with PCID. (b) PCID confusion with new cell installation. (c) Virtual cell for PCID allocation.

and Natural Selection, to solve complex combinatorial problems. Evolutionary-GAs are based on the following major steps:

(1) The initialization step involves mapping the real world problem into a set of strings where individual strings are often termed as chromosomes or genotypes, and the entire set is referred to as population.

(2) The Selection step probabilistically selects a subset of the population based on some fitness value. This fitness value actually determines how good or fit that particular solution is.

(3) The genetic operator crossover probabilistically selects two chromosomes for breeding and obtaining a new "child" chromosome for the next generation. The objective is to create new and diverse solution space. The genetic operator, mutation, on the other hand, probabilistically mutates (changes) a chromosome for obtaining a new chromosomes. Mutation attempts to avoid the possible local optimum values by sudden change (alteration) of chromosomes. While iterating from one generation to the next, the algorithms preserve the fittest (best) chromosome of the present generation [32]. However, mutation is an optional operation depending on the problem domain.

The above-mentioned steps are continued until a satisfying solution is obtained or a maximum number of iterations are completed. With this inspiration from evolutionary-GA, we design BiSON, for an automatic and near-optimal PCID configuration in $5 \mathrm{G}$ UDN.

4.2. Chromosome Initialization. In order to capture and analyze the 5G UDN, containing a myriad of small cells, we introduce a new design of chromosome. Unlike traditional GAs which use multirow chromosomes, our customized
GA uses multicolumn and multirow chromosomes. Every column in a chromosome represents an individual cell in 5G UDN, and every row represents individual PCID allocation status for that cell. The values of each gene in the chromosome represent the "collision" and "confusion" between small cells arising from the configuration. Each gene in the chromosome can have one of the three values: $\{1, \varnothing, \chi\}$. We introduce the following rule for the Initialization of chromosome:

(i) If PCID $\rho$ is assigned to the $i^{\text {th }}$ cell, then set the gene $(\rho, i)$ to $t$.

(ii) If the assignment of PCID $\rho$ to the cell $j$ violates the collision-free or confusion-free constraint, then the gene $(\rho, j)$ is set to $\varnothing$.

(iii) Further, if assigning cell $j$ with the same PCID $\rho$ does not violate any constraint, then set the entry gene $(\rho, j)$ to $\chi$.

First we start with a universally valid allocation by configuring cell $i$ with the PCID $i$. This allocation is always valid, as in any topology, $n$ cells can always be configured by using $n$ unique PCIDs, without violating collision-free and confusion-free constraints. In our chromosome, this relates to setting the diagonal gene $(i, i)=1$. Then for any pair of cells $i$ and $j$, we set $\operatorname{gene}(\rho, j)=\varnothing$, if and only if $\rho=i$ and cell (i) and cell (j) are either neighbors or neighbors' neighbors. All the other remaining entries of row $\rho$ are set to $\chi$. Based on this discussion, we can now mathematically formulate the configuration rules as

$$
\begin{aligned}
& \text { gene }(i, i)=1 ; \\
& \text { gene }(\rho, j) \\
& \quad= \begin{cases}\emptyset, & \text { iff } \rho=i \text { \& collision or confusion; } \\
\chi, & \text { otherwise }\end{cases}
\end{aligned}
$$




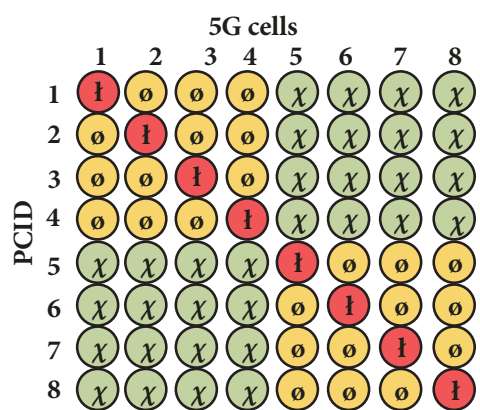

Figure 3: Initial chromosome for topology in Figure 2(a).

As an illustrative example, we represent a topology with 8 small cells, shown in Figure 2(a). Figure 3 represents the initial PCID allocation for the topology in Figure 2(a) where every cell is assigned with a unique PCID. Now, the optimal PCID allocation boils down to the minimization of the number of rows in the chromosome, as each row represents a unique PCID.

4.3. Updating Initial Chromosome. Dynamic installation (e.g., plug-and-play) of new small cells between existing cells might result in conflict in PCID allocations. This raises a significant challenge, as the commercial roll out of $5 \mathrm{G}$ UDN is expected to be carried out gradually in an incremental manner (i.e., gradual installations of new small cells or repeaters over the time). In order to resolve this challenge, we update the initial chromosome to eliminate such configuration conflicts, expected to come up in near future. As shown in Figure 2(a), satisfying initialization steps, initially both cell 4 and cell 8 can be assigned the same PCID. However, as shown in Figure 2(b), a potential problem arises if cell 9 is added in between cells 4 and 8. As a result, either of the two neighbors needs to change their current PCID to meet the "confusion-free" condition for cell 9. In Figure 2(c), we show the process of avoiding such implicit PCID conflicts with the concept of virtual cell or virtual cell cluster following the Update rules, mentioned below:

(i) If gene $(\rho, j)=\chi$ in initial chromosome, then calculate distance $\partial$ between cell $\rho$ and cell $j$.

(ii) Any entry gene $(\rho, j)$ is set to $\varnothing$, if $\partial(i, j) \leq \partial_{r}$, Otherwise, gene $(\rho, j)$ is set to $\chi$,

where $\partial_{r}$ represents the cell radius of specific cell or the size of cell cluster in the unit of cell radius, defined in the SON server. Figure 4 shows the updated chromosome after applying update rules on chromosome in Figure 3. Since cell 4 and cell 8 result in PCID confusion, entry $\chi$ in both $(4,8)$ and $(8,4)$ is now replaced by $\emptyset$.

4.4. Fitness Evaluation and Candidate Solutions. Since the number of rows represents the number of PCID allocation, the lower number of rows represents better solution. In order to minimize the number of rows, we now introduce Fusion Operation (FO) between two rows. Fusion of two rows is

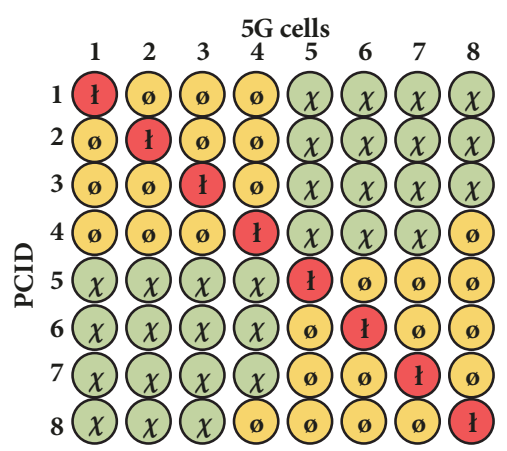

FIGURE 4: Updated chromosome for topology in Figure 2(a).

permitted, only when assigning the same PCID to both cells, which does not create an invalid assignment; i.e., the two cells are not neighbors or neighbors' neighbor. Representing the FO by " $~ W$ ", mathematically we enumerate the FO rules as follows:

(i) Fusion of two rows is not valid if any column in one row is set to $\varnothing$ and the corresponding column in other row is set to $t$.

(ii) If fusion is allowed between two rows, then the set of rules given below determine the resultant row after FO:

$$
\begin{aligned}
& \downarrow \bowtie x= \\
& \imath \bowtie t=\imath \text {; } \\
& ø \bigotimes \chi=ø ; \\
& ø \bigotimes \varnothing=\varnothing ; \\
& \chi \bowtie \chi=\chi \text {; } \\
& \imath \bowtie ø=\text { Illegal; }
\end{aligned}
$$

(iii) Fusion rules mentioned in (i) and (ii) will be repeated for all possible rows in chromosome till fusion is allowed, i.e., until the fusion between the rows results in "Illegal".

Referring to our original example in Figure 2(a), with initial chromosome in Figure 3, a valid set of $\mathrm{FOs}$ is $F O(1,5)$, $F O(2,6), F O(3,7)$, and $F O(4,8)$. Chromosome in Figure 5 demonstrates the result of these FOs. It is clear that the rows of chromosome in Figure 5 cannot support further FO. Thus, we can conclude that the given 5G UDN, (shown in Figure 2(a)), could be optimally configured using four unique PCIDs with the combination of cells $(1,5) ;(2,6)$; $(3,7)$; and $(4,8)$. Furthermore, let us also consider FO over the updated chromosome in Figure 4 with concept of virtual cell in Figure 2(c). Chromosome in Figure 6 delineates the result of similar FO applied over the chromosome in Figure 5. Different from Fusion Operations on chromosome in Figure 5, the same 5G UDN could be allocated with six unique PCIDs, with the combination of cells $(1,5) ;(2,6)$; $(3,7) ; 4$ and 8 . This results from the fact that now cell 4 and 


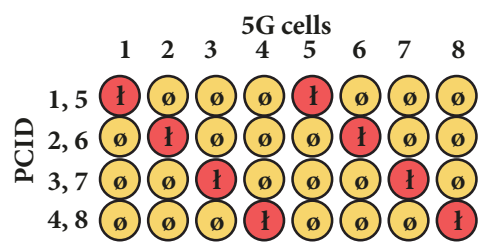

Figure 5: Chromosome after FO in sequence $(1,5),(2,6),(3,7),(4$, 8) without update rule.

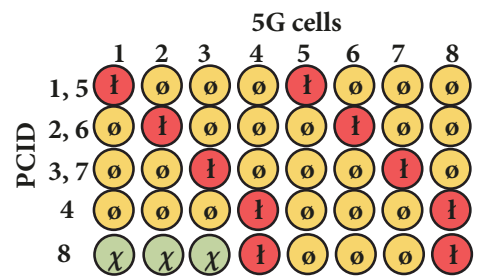

Figure 6: Chromosome after FO in sequence $(1,5),(2,6),(3,7),(4$, 8) with update.

cell 8 are configured with unique $\mathrm{PCID}$, as $F O(4,8)$ is not permitted according to FO rules. At the end of the FOs, the remaining set of rows provides a viable candidate solution for the PCID configuration problem.

\section{BiSON: Biologically Inspired Approach for Optimal PCID Allocation}

In this section, we introduce our proposed near-optimal PCID configuration algorithm, i.e., BiSON. Subsequently, we also provide two different extensions of BiSON for dynamic introduction and removal of $5 \mathrm{G}$ small cells.

5.1. Randomized BiSON Algorithm. The flow of BiSON is demonstrated in Figure 7. The BiSON algorithm follows the steps as mentioned below:

(1) Based on the Neighbor Relation Table (NRT) of ANR in SON server, a chromosome $C M_{\text {init }}$ can be initialized using initialization rules, and followed by Update rules, as discussed in Section 4. Let $r$ represent the total number of rows in $C M_{\text {init }}$.

(2) Let any chromosome $\mathfrak{J}_{i}$ represent $r$ rows in $C M_{\text {init }}$ in any specific order. An initial generation, of size $\lambda$, contains randomly generated permutations of the set of $r$ rows in the initial chromosome mathematically, if $\overrightarrow{\mathfrak{\Im}}(0)$ denote the initial generation, then,

$$
\begin{aligned}
\overrightarrow{\mathfrak{I}}(0)=\left\{\mathfrak{I}_{1}(0), \mathfrak{J}_{2}(0), \ldots, \mathfrak{I}_{\lambda}(0)\right\}, & \\
& \text { where } \mathfrak{J}_{i}(0)=\left\{\ldots R_{i}(0), \ldots\right\} \&\left|\mathfrak{\Im}_{i}(0)\right|=r
\end{aligned}
$$

(3) At each iteration $t$ do the following with the current generation:

(a) Genetic Operation ( $\mathscr{G})$ : Probabilistically select two rows $R_{p}$ and $R_{q}$ from every chromosome $\mathfrak{\Im}_{i}$.

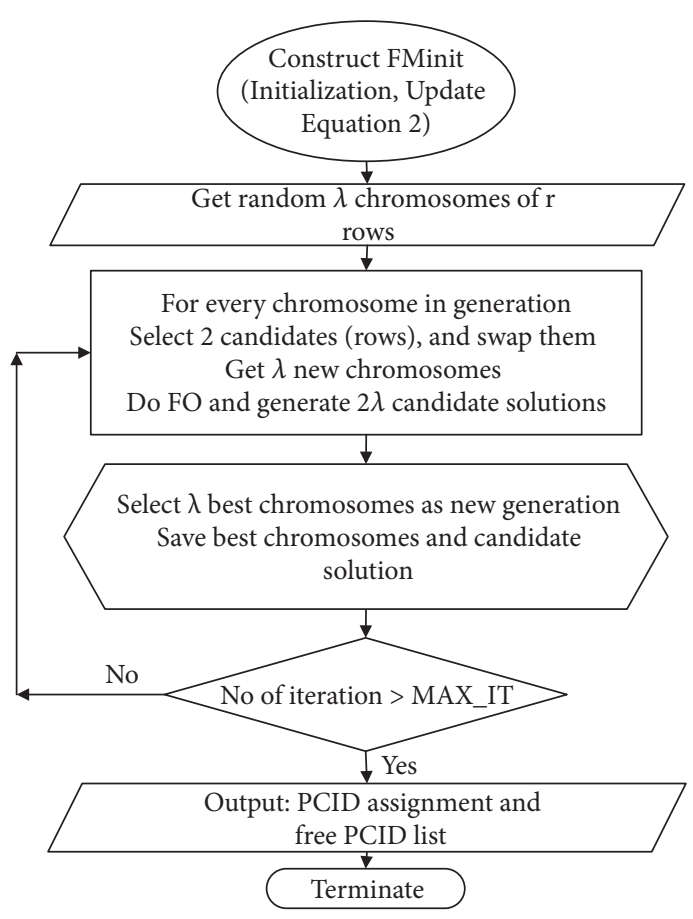

Figure 7: Proposed BiSON algorithm.

Generate $\lambda$ offspring by swapping the selected rows. Now, the generation has $2 \lambda$ chromosome.

(b) Fitness Evaluation: Perform FO over the sequence of $r$ rows belonging to each permutation $\mathfrak{J}_{i}$ and obtain $2 \lambda$ candidate solutions. Evaluate the fitness value of each candidate solution. The fitness function is formulated as $f\left(\mathfrak{\Im}_{i}(t)\right)=1 /\left|\mathfrak{\Im}_{i}(t)\right|$, where $\left|\Im_{i}(t)\right|$ represents the cardinality of set $\mathfrak{\Im}_{i}(t)$. Since the number of rows represents the number of PCID allocation, the lower number of rows represents better solution.

(c) Reproduction ( $\mathfrak{R})$ : Probabilistically, select $\lambda$ best individual (chromosome) for the new generation $\overrightarrow{\mathfrak{\Im}}(t+1)$. Mathematically, we can say that

$$
\overrightarrow{\boldsymbol{\Im}}(t+1)=\mathfrak{R}(\mathscr{G}(\overrightarrow{\boldsymbol{\Im}}(t)))
$$

(d) Store optimal permutation $\mathfrak{\Im}_{o p t}(t+1)$ from the set $\overrightarrow{\mathfrak{\Im}}(t+1)$ and optimal individual $\mathfrak{\Im}_{o p t}(t)$.

(4) Stopping Criteria: The stopping criteria are number of maximum iteration $T_{\max }$. If $T_{\max }$ number of iteration is complete, stop the execution, else repeat step (3).

In step (3), the algorithm gradually explores the search spaces and stores the $\lambda$ best chromosome. Thus, at every iteration, the algorithm tries to improve its candidate solutions. At the end of the algorithm, the chromosome $\mathfrak{\Im}_{\text {opt }} \in \overrightarrow{\mathfrak{\Im}}\left(T_{\text {max }}\right)$, with the least number of rows, is selected as the near-optimal PCID allocation strategy. The number of rows, $\mathscr{R}_{\text {opt }} \in$ 


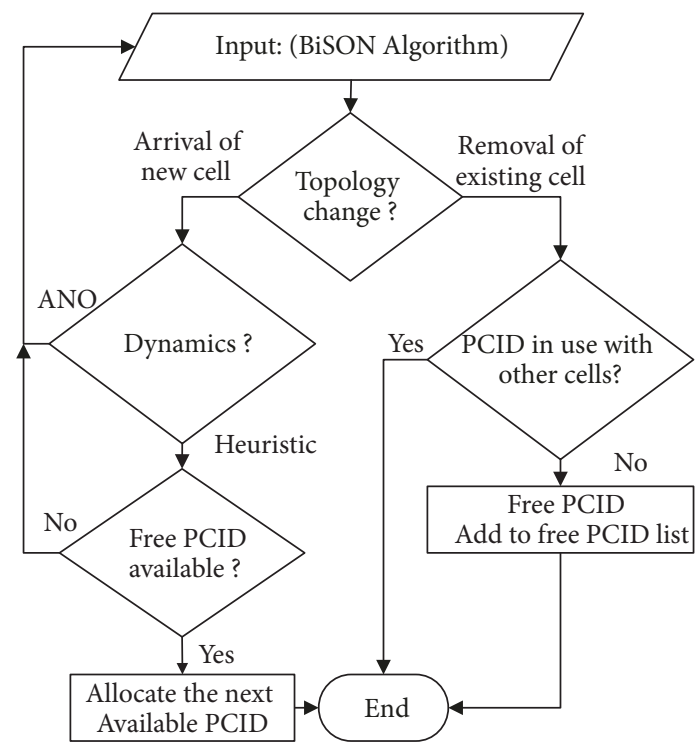

FIgURe 8: Dynamic PCID allocation algorithm.

$\Im_{o p t}$, represents the number of PCIDs needed to allocate in the 5G UDN with the collision-free and confusion-free constraints.

5.2. Dynamic PCID Allocation. New $5 \mathrm{G}$ small cells are added to and removed from the industrial network dynamically. Hence, the BiSON algorithm needs periodic reexecution to accept the dynamic inclusion of small cells. We present two distinct extensions which offer compromise between optimal solution and computation complexity. Figure 8 shows the flowchart for dynamic PCID allocation algorithm.

(1) Always near-optimal (ANO). The topology of the 5G UDN changes with the addition or removal of single or multiple cells. The BiSON algorithm is executed to obtain the new near-optimal PCID allocation for the new topology. This procedure always provides a new near-optimal PCID allocation. However, it also suffers from more computational overhead, arising from the frequent execution of the entire algorithm.

(2) Heuristic. Initial execution of BiSON during network installation results in specific near-optimal PCID allocation with $\mathscr{R}_{\mathrm{OPT}}$ different PCIDs. After the initial execution, $\left(\wp-\mathscr{R}_{\mathrm{OPT}}\right)$ PCIDs will remain unallocated. When new cells are added, the algorithm allocates a new PCID from this set of $\left(\wp-\mathscr{R}_{\mathrm{OPT}}\right)$ unallocated PCIDs, until this set becomes empty. When there is no free PCID, BiSON is re-executed. Comparing with "ANO", the heuristic provides suboptimal solution, at the cost of lower computational overhead.

On the other hand, the removal dynamics of any cell is quite simple. During removal of cells, both "ANO" and "Heuristic" algorithm check for another small cell with the same PCID.
If such a cell does not exist, both approaches add the PCID to the free PCID list.

\section{Modeling and Convergence Analysis of BiSON}

In this section, we discuss the stochastic behavior of BiSON algorithm, show its convergence to optimality and subsequently, and analyze asymptotic behavior.

6.1. Markov Chain Modeling. The BiSON algorithm, mentioned in Section 5.1, shows that at any iteration $t$, the chromosome-set $\overrightarrow{\mathfrak{\Im}}(t)$ depends only on the chromosome-set of the previous iteration $\overrightarrow{\mathfrak{\Im}}(t-1)$. Hence, mapping every chromosome-set to the corresponding state, the underlying process could be modeled as a Discrete Time Markov Chain $[33,34]$, with state space $\Psi$. If $\operatorname{Pr}$ represents the corresponding state probability, then mathematically we can say $\operatorname{Pr}[\overrightarrow{\boldsymbol{\Im}}(t) \mid \overrightarrow{\boldsymbol{\Im}}(0), \ldots, \overrightarrow{\boldsymbol{\Im}}(t-1)]=\operatorname{Pr}[\overrightarrow{\boldsymbol{\Im}}(t) \mid \overrightarrow{\boldsymbol{\Im}}(t-1)]$. Defining $\Psi_{\mathrm{OPT}}$ as the globally optimal state set, if any current optimal chromosome $\mathfrak{J}_{i}(t)$, at iteration $t$, is the globally optimal chromosome (fittest), then $\mathfrak{\Im}_{i}(t) \in \Psi_{\mathrm{OPT}}$. Following the stopping criteria of BiSON algorithm, as mentioned in step (4), $\mathfrak{\Im}_{i}(t+1)=\mathfrak{\Im}_{i}(t)$ and $\overrightarrow{\mathfrak{\Im}}(t+1) \in \Psi_{\mathrm{OPT}}$. Thus, mathematically we get the following relation:

$$
\operatorname{Pr}\left[\overrightarrow{\mathfrak{\Im}}(t+1) \notin \Psi_{O P T} \mid \overrightarrow{\boldsymbol{\Im}}(t) \in \Psi_{O P T}\right] \sim 0
$$

Based on (6), we can state that $\overrightarrow{\boldsymbol{\Im}}(t)$ is an absorbing Markov process, with subspace $\Psi_{O P T}$ as an absorbing state subset. Now, let us assume $P_{\mathscr{G}}$ and $P_{\mathfrak{R}}$ represent the probability of genetic operation and reproduction operation, mentioned in step (3) of BiSON algorithm. As the genetic operation randomly selects any two row identifiers $\left(R_{p}, R_{q}\right)$ for each of the $\lambda$ chromosome (each chromosome containing $r$ rows), mathematically we can say

$$
P_{\mathscr{G}}=\prod_{i=1}^{\lambda} \frac{1}{r(r-1)}=\frac{1}{[r(r-1)]^{\lambda}}
$$

Furthermore, as the reproduction operation probabilistically selects $\lambda$ as the best chromosome from the set of $2 \lambda$ chromosomes, we can say

$$
P_{\Re}=\prod_{i=1}^{\lambda} \frac{f\left(\mathfrak{\Im}_{i}\right)}{\sum_{j=1}^{2 \lambda} f\left(S_{j}\right)}=\frac{\prod_{i=1}^{\lambda} f\left(\mathfrak{\Im}_{i}\right)}{\left[\sum_{j=1}^{2 \lambda} f\left(S_{j}\right)\right]^{\lambda}}
$$

Let $\mathbb{M}$ represent the state transition probability matrix associated with BiSON, then $\mathbb{M}$ can be decomposed as a product of stochastic matrices: $\mathbb{M}=\mathscr{G} \cdot \mathfrak{R}$, where $\mathscr{G}$ and $\mathfrak{R}$ contain probability elements $P_{\mathscr{G}} \geq 0$ and $P_{\Re} \geq 0$, respectively. Thus, we can say that $\mathbb{M}$ is a positive and primitive matrix [34]. At this point we can say the BiSON algorithm with probabilities mentioned above is an ergodic Markov Chain; i.e., there exists a unique limit distribution with nonzero probability for the states of the chain to be in any state at any time, regardless of the initial distribution. 
6.2. Convergence Analysis. Before analyzing the convergence of BiSON algorithm, we first define convergence as follows. Let $C_{n}, \forall n \geq 0$, be a sequence of random variable. We call that $C_{n}$ converges to $\mathbb{C}$ if it satisfies the following:

$$
\lim _{n \rightarrow \infty} \operatorname{Pr}\left[C_{n} \in \mathbb{C}\right]=1
$$

From above definitions and modeling of BiSON algorithm, we can conclude that the stochastic process associated with $\overrightarrow{\mathfrak{\Im}}(t)$ is reducible, and the optimal state set $\mathbb{C}$ is a closed set with aperiodic and irreducible properties. In order to analyze the convergence of Markov Chain $\overrightarrow{\mathfrak{\Im}}(t)$, we use the following important convergence definition [33]:

For any reducible stochastic matrix $\mathbb{M}$, the following relation holds:

$$
\lim _{j \rightarrow \infty} \mathbb{M}^{j}=\mathbb{M}^{\infty}=1_{m \times 1}^{\prime} \cdot \pi^{\infty}
$$

which is a stable stochastic matrix where $\pi^{\infty}=\left(\pi_{1}, \ldots, \pi_{m}\right)$ and $\sum_{i=1}^{m} \pi_{i}=1$. If $\operatorname{Pr}[\overrightarrow{\mathfrak{\Im}}(t)]$ is the probability distribution of $\overrightarrow{\mathfrak{s}}(t)$, then from the definition of Markov Chain we can obtain $\operatorname{Pr}[\overrightarrow{\boldsymbol{\Im}}(t)]=\operatorname{Pr}[\overrightarrow{\boldsymbol{\Im}}(0)] \cdot \mathbb{M}^{t}$. From the definition of convergence, we can say that there exists a stable distribution $\pi^{\infty}$ which satisfies $\left|\mathbb{M}^{t}-1^{\prime} \pi^{\infty}\right| \longrightarrow 0$. The values of $\pi^{\infty}$ corresponding to $\Psi \mid \Psi_{O P T}$ approach zero, thus resulting in the following relation:

$$
\begin{aligned}
& \operatorname{Pr}\left[\overrightarrow{\mathfrak{\Im}}(t) \in \Psi \mid \Psi_{O P T}\right] \sim 0 \Longrightarrow \\
& \lim _{t \rightarrow \infty} \operatorname{Pr}\left[\overrightarrow{\mathfrak{\Im}}(t) \in \Psi_{O P T}\right] \sim 1
\end{aligned}
$$

Based on this analysis, now we can conclude that the BiSON algorithm converges to the optimal state set $\Psi_{O P T}$.

6.3. Convergence Speed. In order to analyze the convergence speed of BiSON, we first state the definition of a minorization condition [33] for the Markov Chain: A minorization condition for a Markov Chain $\{\overrightarrow{\boldsymbol{\Im}}(t), t \geq 0\}$ is a pair $(\alpha, \beta,(\cdot))$ such that $P(\overrightarrow{\mathfrak{\Im}}(t), X) \geq \alpha \beta(X), \forall \overrightarrow{\mathfrak{\Im}} \in \Psi$ and $X \subset \Psi$, where $\alpha$ is a positive real number and $\beta$ is a probability distribution on $\Psi$. With a stationary distribution $P_{\text {stat }}$, given any initial distribution $\left|P(t)-P_{\text {stat }}\right| \leq(1-\alpha)^{t}[33]$.

Considering the BiSON algorithm, we can say that the number of chromosomes of the corresponding state of associated Markov Chain $\{\overrightarrow{\boldsymbol{\Im}}(t), t \geq 0\}$ is $\lambda$, and each chromosome $\mathfrak{J}_{i}$ consists of $r$ rows in any order. As every element of any row in the generation can be either of three values $(1, \varnothing, \chi)$, if $\ell$ represents the current number of entries of every row, then the total possible number of permutations in $\Psi$ is $3^{\ell r \lambda}$.
Referring to (7) and (8) from Section 6.1, we can have the following relation:

$$
\begin{aligned}
P_{\mathscr{G}} & =\frac{1}{[r(r-1)]^{\lambda}} \geq \frac{1}{r^{r \lambda}}, \\
\text { and } P_{\mathfrak{R}} & =\frac{\prod_{i=1}^{\lambda} f\left(\mathfrak{\Im}_{i}\right)}{\left[\sum_{j=1}^{2 \lambda} f\left(\mathfrak{\Im}_{j}\right)\right]^{\lambda}} \geq\left[\frac{f\left(\mathfrak{\Im}_{l}\right)}{\sum_{j=1}^{2 \lambda} f\left(\mathfrak{\Im}_{j}\right)}\right]^{\lambda},
\end{aligned}
$$

where $f\left(\mathfrak{\Im}_{l}\right)$ represents the chromosome $S$ with lowest $f(S)$. Now if we define $\alpha=\left[\left(3^{\ell} / r\right)^{r}\left(f\left(\mathfrak{\Im}_{l}\right) / \sum_{j=1}^{2 \lambda} f\left(\mathfrak{\Im}_{j}\right)\right)\right]^{\lambda}$, and $\beta(X)=3^{-\ell r \lambda} \cdot|X|$, where $|X|$ represents the cardinality of $X$, then for any $(t)$ and $X \subset \Psi$, we can say that

$$
\begin{aligned}
P(\overrightarrow{\boldsymbol{J}}, X) & =\sum_{\overrightarrow{\boldsymbol{V}} \in X} P(\overrightarrow{\mathfrak{J}}, \overrightarrow{\boldsymbol{V}}) \geq\left[\frac{1}{r^{r \lambda}}\right]\left[\frac{f\left(\mathfrak{\Im}_{l}\right)}{\sum_{j=1}^{2 \lambda} f\left(\mathfrak{\Im}_{j}\right)}\right]^{\lambda} \\
& =\alpha \beta(X)
\end{aligned}
$$

Hence, we can conclude that $(\alpha, \beta(\cdot))$ defined above is a minorization condition for the Markov Chain representation of BiSON. Thus, based on minorization condition definition, we can conclude the following on the speed of convergence of BiSON: the Markov Chain corresponding to the BiSON algorithm, with a stationary probability distribution $P$ and probability distribution $P(t)$ of $t^{\text {th }}$ generation, satisfies the following equation:

$$
|P(t)-P| \leq\left(1-\left[\left(\frac{3^{\ell}}{r}\right)^{r}\left(\frac{f\left(\mathfrak{\Im}_{l}\right)}{\sum_{j=1}^{2 \lambda} f\left(\mathfrak{J}_{j}\right)}\right)\right]^{\lambda}\right)^{t}
$$

Based on the above equation, we can easily infer that the convergence speed of BiSON increases with increasing value of $f\left(\mathfrak{I}_{l}\right)$ and reduces with increasing values of $\lambda$ and $r$.

\section{Simulation Experiments and Results}

In this section, we first introduce our NS3-based network simulator and describe the simulation scenario. Subsequently, we discuss our simulation results and present the efficiency and complexity of proposed algorithms.

\subsection{Simulation Parameters}

(1) We consider a $13,400 \mathrm{~m}^{2}$ dense urban environment with 120 macrocells, each having a radius of $400 \mathrm{~m}$.

(2) The 5G small cells have radius $\sim 50 \mathrm{~m}$ and are deployed in a random fashion. The number of small cell deployment gradually increased from 1,000 to 12,000 .

(3) The BiSON algorithm runs in the SON server and dynamically allocates PCID to small cells.

(4) We have used dense urban 5G channel model specified in [25] and the RF parameters from Samsung's 
TABLE 3: Major experimental and simulation parameters.

\begin{tabular}{lcc}
\hline & 5G Radio Access Network Models [24, 25] & \multicolumn{2}{c}{} \\
\hline Penetration loss & & 20dB \\
\hline Attenuation factor & Macro and Small Cell Parameters & 3.6 \\
\hline Path loss compensation & & $20 \%$ \\
\hline & & $50 \%$ \\
\hline RRH switch-off threshold & Additional Simulation Parameter \\
\hline RRH switch-on threshold & & 600 \\
\hline & & 100 \\
\hline Number of UEs per macro cell & \\
\hline Number of UEs per small cell & Random waypoint model [26] \\
\hline UE mobility model & 30 days \\
\hline Simulation duration &
\end{tabular}

5G field test [24] which states an RRH's transmission power of $31 \mathrm{dBm}$, idle power of $5 \mathrm{dBm}$, penetration loss of $25 \mathrm{~dB}$, shadowing deviation of 0.8 , mobile UE's maximum power of $20 \mathrm{dBm}$ in $27.925 \mathrm{GHz}$ frequency band, and $520 \mathrm{MHz}$ channel bandwidth. Table 3 highlights other $5 \mathrm{G}$ networks, and radio parameters used in our simulations.

(5) We also include multistory buildings where small cells are deployed with arbitrary overlapping of one over another.

(Data used for the manuscript is available at http://www .abhishekroy.info/slides.html)

7.2. Results and Discussion. We have simulated our algorithm with NS-3 based system simulator, compared our BiSON simulation result with Permutation Merge Model with Guided Random Search (PMM-GRS) algorithm [5], and distributed graph coloring based PCID allocation [3]. Figure 9(a) delineates the competitive PCID allocation using BiSON, PMM-GRS and graph coloring based assignment without confusion. The BiSON algorithm exhibits an average of $53 \%$ and $55 \%$ enhancement in PCID allocation more than the PMM-GRS, and the graph coloring based PCID assignment strategy, respectively. Using 504 PCIDs, graph coloring based PCID allocation and PMM-GRS can allocate a multiple of $\sim 4,500$ and $\sim 5,000$ cells, respectively, under the same server, whereas BiSON can support up to 11,000 cells. Using BiSON, only 301 unique PCID can configure up to 7,000 cells including macrocells and small cells. Figure 9(b) presents the execution time of BiSON, compared to graph coloring based PCID assignment and PMM-GRS approach. Proposed BiSON algorithm can find a near-optimal solution in $70 \sim 130$ s for $3000 \sim 7000$ cells. As compared to BiSON, PMM-GRS needs 20\% additional execution time for a higher number of $5 \mathrm{G}$ small cells while graph coloring based PCID allocation without confusion takes $\sim 30 \%$ more execution time than the BiSON.

BiSON uses two solution approaches: Heuristic and ANO for both addition and removal of new cells. Comparative PCID allocation for both strategies with 500 base stations is shown in Figure 9(c). At the arrival of every new base station, ANO strategy finds an optimal PCID allocation at each time. On the other hand, the heuristic approach computes the nearoptimal PCID allocation once and then continues assigning new PCID to the new base stations until all the available PCID get allocated. After all the PCIDs are allocated, it re-executes the algorithm and finds a new near-optimal PCID allocation. Figure $9(\mathrm{~d})$ shows the number of operations performed by both strategies. The heuristic approach shows $~ 95$ times better performance than the ANO approach in regard to computational overhead.

Dynamics of future confusion evaluation and avoidance according to update rules are presented in Figure 9(e). With more than 80 PCID reserved, future confusion is reduced to almost zero. BiSON can estimate all the possible virtual cells and allocate PCIDs on their arrivals. If less than 10 PCIDs are reserved, the number of confusion can be up to $\sim 180$.

Figure 9(f) presents the trade-off between the number of initial cells supported with the number of future confusions. As shown in this figure, with less than 11,500 small cells, BiSON encounters no future confusion. This is a direct consequence of update rule to reserve some PCIDs for future cells. With a large number of initial cells, the number of reserved PCIDs for future dynamic addition is small, leading to early violation of confusion constraint. For more than 12,000 cells, BiSON incurs 80 future confusions.

Figure 10 shows the convergence speed of proposed BiSON with respect to the parameters $\lambda$ and $r$. With low value of $\lambda$ and $r$, the proposed algorithm completes in less than 10s. With increasing $\lambda$ and $r$, the convergence time increases. BiSON's convergence time increases up to 550 s in a deployment scenario where $\lambda=50$ and $r=500$ in a dense urban environment.

\section{Conclusion}

In this paper, we have proved that the optimal PCID allocation is NP-hard problem. Subsequently, we proposed Bioinspired Self-Organizing Network (BiSON) algorithm to find a near-optimal PCID allocation for 5G ultradense network. 


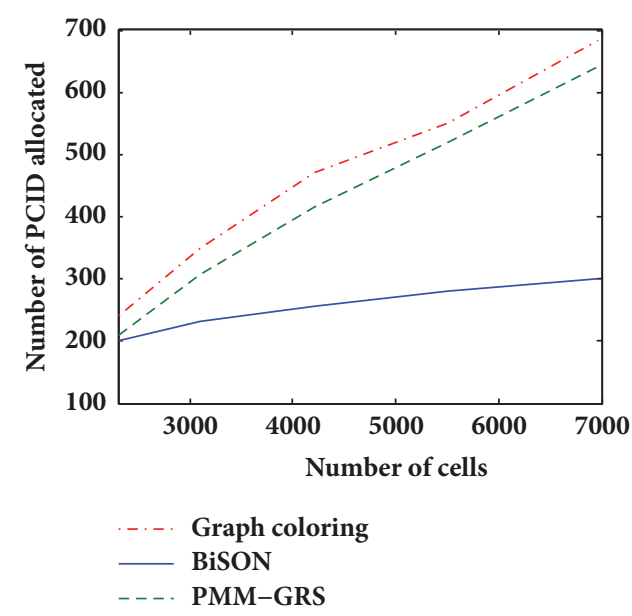

(a)
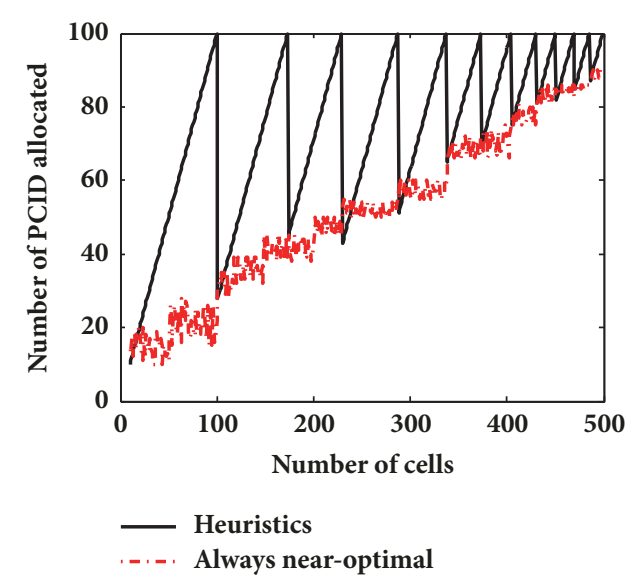

(c)

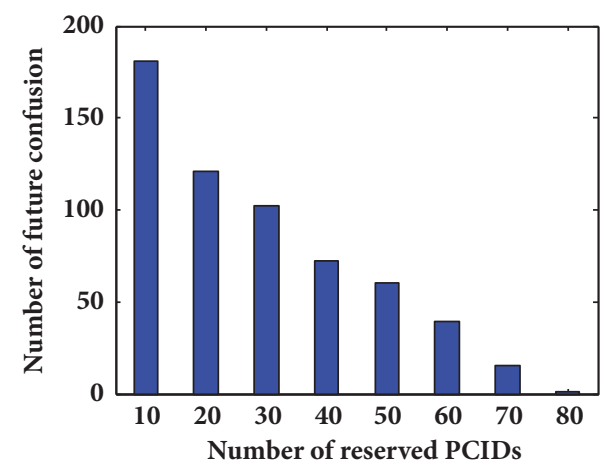

(e)

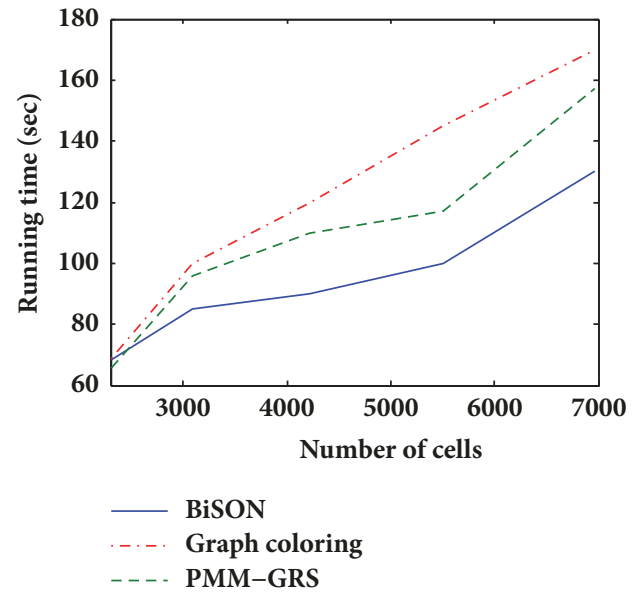

(b)

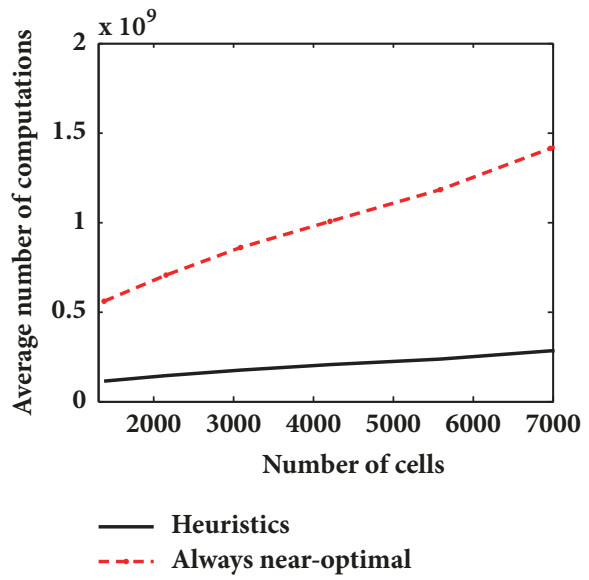

(d)

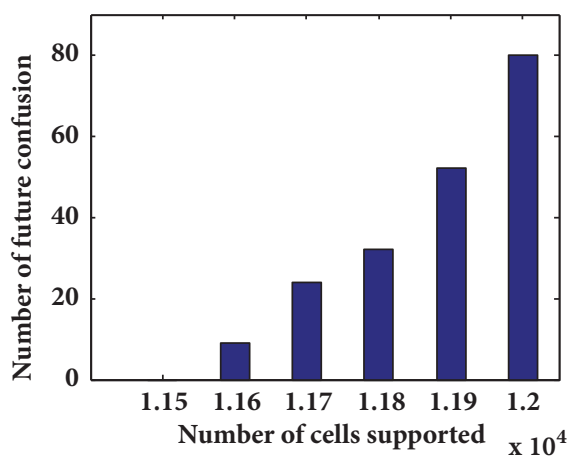

(f)

FIgURE 9: (a) Near-optimal PCID allocation, (b) comparative execution time, (c) PCID allocation dynamics, (d) comparative computational overhead, (e) confusion and PCID allocation, (f) confusion and no. of cells supported.

We discussed dynamics of near-optimal PCID allocation strategy and described two distinct approaches to support dynamic addition and removal of small cells in the 5G ultradense network. We analyzed the proposed algorithm and showed its convergence to an upper bound. Simulation results in an ultradense deployment demonstrate that our strategy can improve auto-configuration by $53 \% \sim 55 \%$ more than the previous approaches within feasible time and acceptable computational overhead.

\section{Data Availability}

Data used in the manuscript is available at http://www .abhishekroy.info/slides.html. 


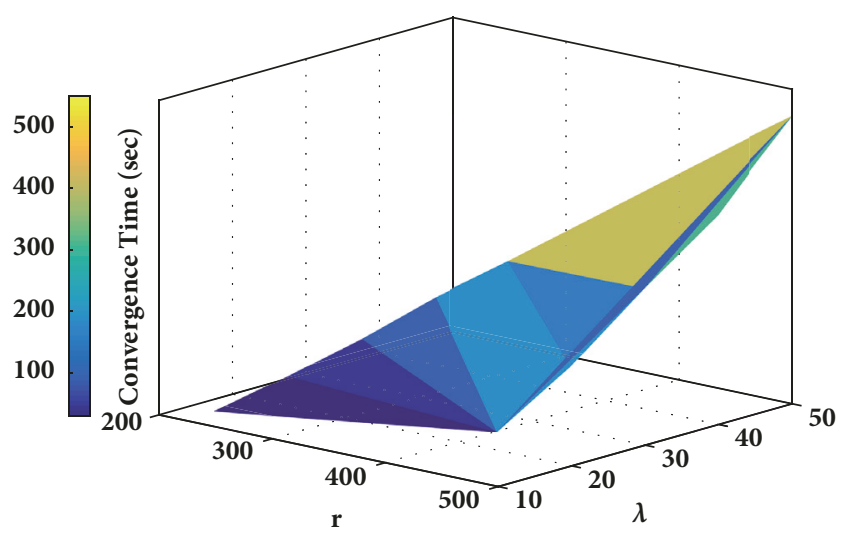

FIgURE 10: BiSON convergence.

\section{Conflicts of Interest}

The authors declare that they have no conflicts of interest.

\section{Acknowledgments}

This research is supported by Basic Science Research Program through the National Research Foundation of Korea (NRF) funded by the Ministry of Education (NRF2016R1D1A1B03935633).

\section{References}

[1] N. Bhushan, J. Li, D. Malladi et al., "Network densification: the dominant theme for wireless evolution into 5G," IEEE Communications Magazine, vol. 52, no. 2, pp. 82-89, 2014.

[2] C. G. Aliu, "A Survey of Self Organisation in Future Cellular Networks," IEEE Commun. Surveys Tutorials, vol. 15, no. 1, pp. 336-361, 2012.

[3] F. Ahmed et al., "Distributed Graph Coloring for SelfOrganization in LTE Networks," Journal of Electrical and Computer Engineering, vol. 2010, Article ID 402831, 10 pages, 2010.

[4] M. Krichen, D. Barth, and O. Marce, "Performances evaluation of different algorithms for PCIs self configuration in LTE," in Proceedings of the 2012 18th IEEE International Conference on Networks, ICON 2012, pp. 197-203, Singapore, December 2012.

[5] N. Saxena, A. Roy, H. Kim, and J.-J. Won, "Auto-configuration of physical cell ID in LTE femtocellular systems using self organizing networks," Wireless Networks, vol. 20, no. 5, pp. 11071120, 2013.

[6] A. Engels, M. Reyer, X. Xu, R. Mathar, J. Zhang, and H. Zhuang, "Autonomous self-optimization of coverage and capacity in LTE cellular networks," IEEE Transactions on Vehicular Technology, vol. 62, no. 5, pp. 1989-2004, 2013.

[7] M. Goonewardena, H. Akbari, W. Ajib, and H. Elbiaze, "On minimum-collisions assignment in heterogeneous selforganizing networks," in Proceedings of the 2014 IEEE Global Communications Conference, GLOBECOM 2014, pp. 46654670, December 2014.

[8] M. Ahmed, S.-G. Yoon, S. Jeong, and S. Bahk, "Geo-location based self-organization scheme for femtocell networks," in Proceedings of the 2013 International Conference on Information and Communication Technology Convergence, ICTC 2013, pp. 535-539, Jeju, South Korea, October 2013.

[9] L. M. Abdullah, M. Dani Baba, and S. G. Ali, "A novel scheme to resolve PCI conflicts and assignment problems in LTE-femtocell networks," in Proceedings of the 2013 IEEE 3rd International Conference on System Engineering and Technology (ICSET), pp. 109-112, Shah Alam, Malaysia, August 2013.

[10] D. Palacios, I. de-la-Bandera, A. Gomez-Andrades, L. Flores, and R. Barco, "Automatic feature selection technique for next generation self-organizing networks," IEEE Communications Letters, vol. 22, no. 6, pp. 1272-1275, 2018.

[11] M. Sun, H. Qian, K. Zhu, D. Guan, and R. Wang, "Ensemble Learning and SMOTE Based Fault Diagnosis System in SelfOrganizing Cellular Networks," in Proceedings of the GLOBECOM 2017 - 2017 IEEE Global Communications Conference, pp. 1-6, Singapore, December 2017.

[12] P. V. Klaine, M. A. Imran, O. Onireti, and R. D. Souza, "A Survey of Machine Learning Techniques Applied to Self Organizing Cellular Networks," IEEE Communications Surveys \& Tutorials, vol. 19, no. 4, 2017.

[13] S. Bitam, A. Mellouk, and S. Zeadally, "Bio-inspired routing algorithms survey for vehicular ad hoc networks," IEEE Communications Surveys \& Tutorials, vol. 17, no. 2, pp. 843-867, 2015.

[14] F. Dressler and O. Akan, "Bio-inspired networking: From theory to practice," IEEE Communications Magazine, vol. 48, no. 11, pp. $176-183,2010$.

[15] F. Dressler and O. B. Akan, "A survey on bio-inspired networking," Computer Networks, vol. 54, no. 6, pp. 881-900, 2010.

[16] Y. Liu, M. Tao, B. Li, and H. Shen, "Optimization framework and graph-based approach for relay-assisted bidirectional OFDMA cellular networks," IEEE Transactions on Wireless Communications, vol. 9, no. 11, pp. 3490-3500, 2010.

[17] W. Rankothge, F. Le, A. Russo, and J. Lobo, “Optimizing resource allocation for virtualized network functions in a cloud center using genetic algorithms," IEEE Transactions on Network and Service Management, vol. 14, no. 2, pp. 343-356, 2017.

[18] E. Amaldi, A. Capone, and F. Malucelli, "Radio planning and coverage optimization of $3 \mathrm{G}$ cellular networks," Wireless Networks, vol. 14, no. 4, pp. 435-447, 2008.

[19] S. K. Biswash, S. Nagaraj, M. Sarkar, and N. R. Narra, "Bee System Based Base Station Cooperation Technique for Mobile Cellular Networks," Wireless Personal Communications, vol. 92, no. 3, pp. 1193-1220, 2016.

[20] O. M. Alia and A. Al-Ajouri, "Maximizing Wireless Sensor Network Coverage with Minimum Cost Using Harmony Search Algorithm," IEEE Sensors Journal, vol. 17, no. 3, pp. 882-896, 2017.

[21] H. Byun, S. Son, and S. Yang, "Biologically inspired node scheduling control for wireless sensor networks," Journal of Communications and Networks, vol. 17, no. 5, pp. 506-516, 2015.

[22] S. Barbarossa and G. Scutari, "Bio-inspired sensor network design," IEEE Signal Processing Magazine, vol. 24, no. 3, pp. 2635, 2007.

[23] F. Gómez Mármol and G. Martínez Pérez, "Providing trust in wireless sensor networks using a bio-inspired technique," Telecommunication Systems, vol. 46, no. 2, pp. 163-180, 2011.

[24] W. Roh, J.-Y. Seol, J. Park et al., "Millimeter-wave beamforming as an enabling technology for $5 \mathrm{G}$ cellular communications: theoretical feasibility and prototype results," IEEE Communications Magazine, vol. 52, no. 2, pp. 106-113, 2014. 
[25] T. S. Rappaport, F. Gutierrez, E. Ben-Dor, J. N. Murdock, Y. Qiao, and J. I. Tamir, "Broadband millimeter-wave propagation measurements and models using adaptive-beam antennas for outdoor Urban cellular communications," IEEE Transactions on Antennas and Propagation, vol. 61, no. 4, pp. 1850-1859, 2013.

[26] E. Hyytiä and J. Virtamo, Random Waypoint Mobility Model in Cellular Networks, vol. 13, Springer Wireless Netw.s, 2007.

[27] A. Imran and A. Zoha, "Challenges in 5G: how to empower SON with big data for enabling 5G," IEEE Network, vol. 28, no. 6, pp. 27-33, 2014.

[28] E. J. Khatib, R. Barco, A. Gómez-Andrades, and I. Serrano, "Diagnosis Based on Genetic Fuzzy Algorithms for LTE SelfHealing," IEEE Transactions on Vehicular Technology, vol. 65, no. 3, pp. 1639-1651, 2016.

[29] M. R. Garey and D. S. Johnson, Computers and Intractability: A Guide to the Theory of NP-Completeness, W. H. Freeman Publishers, San Francisco, Calif, USA, 1979.

[30] D. B. West, Introduction to Graph Theory, Prentice Hall Publications, Upper Saddle River, NJ, USA, 2000.

[31] S. Even, Algorithmic Combinatorics, Collier-Macmillan, London, UK, 1973.

[32] A. E. Eiben and J. E. Smith, Introduction to Evolutionary Computing, Natural Computing Series, Springer, 2007.

[33] J. S. Rosenthal, "Minorization conditions and convergence rates for Markov chain Monte Carlo," Journal of the American Statistical Association, vol. 90, no. 430, pp. 558-566, 1995.

[34] G. Rudolph, "Convergence analysis of canonical genetic algorithms," IEEE Transactions on Neural Networks and Learning Systems, vol. 5, no. 1, pp. 96-101, 1994. 


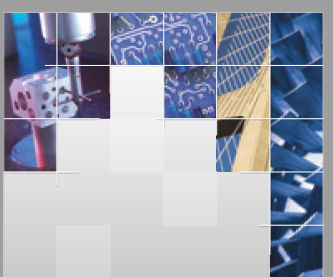

\section{Enfincering}
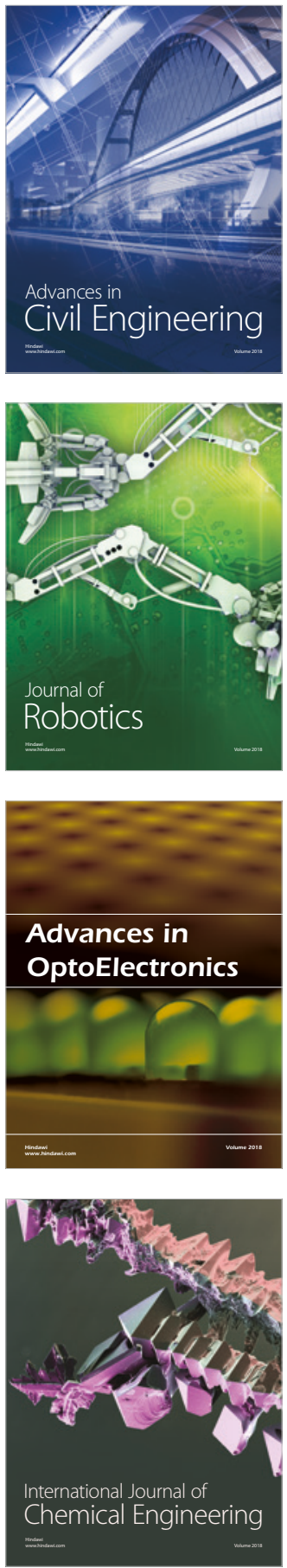

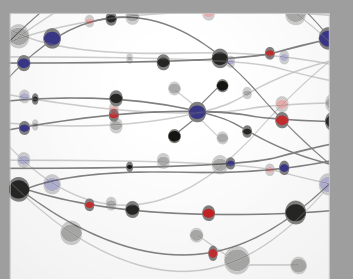

\section{Rotating \\ Machinery}

The Scientific World Journal

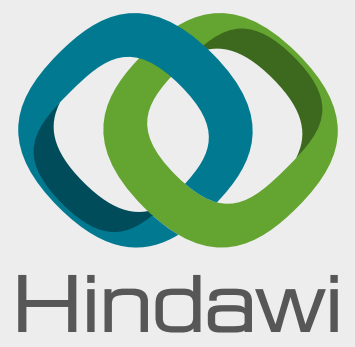

Submit your manuscripts at

www.hindawi.com
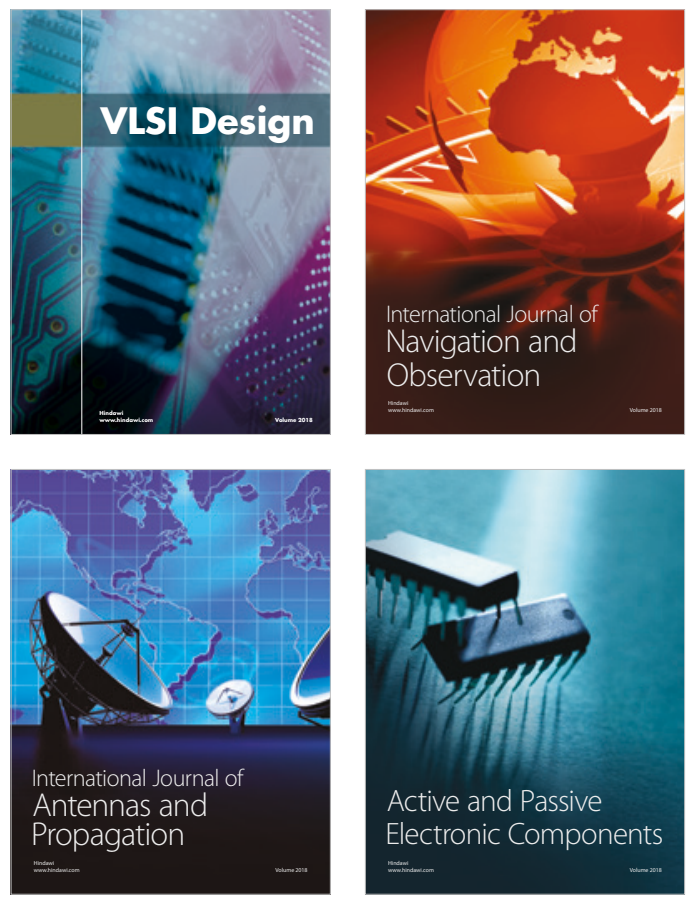
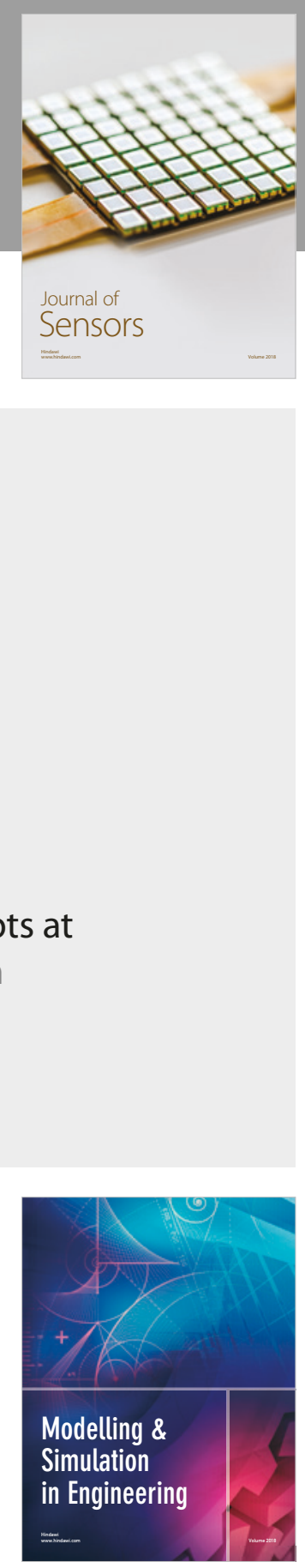

\section{Advances \\ Multimedia}
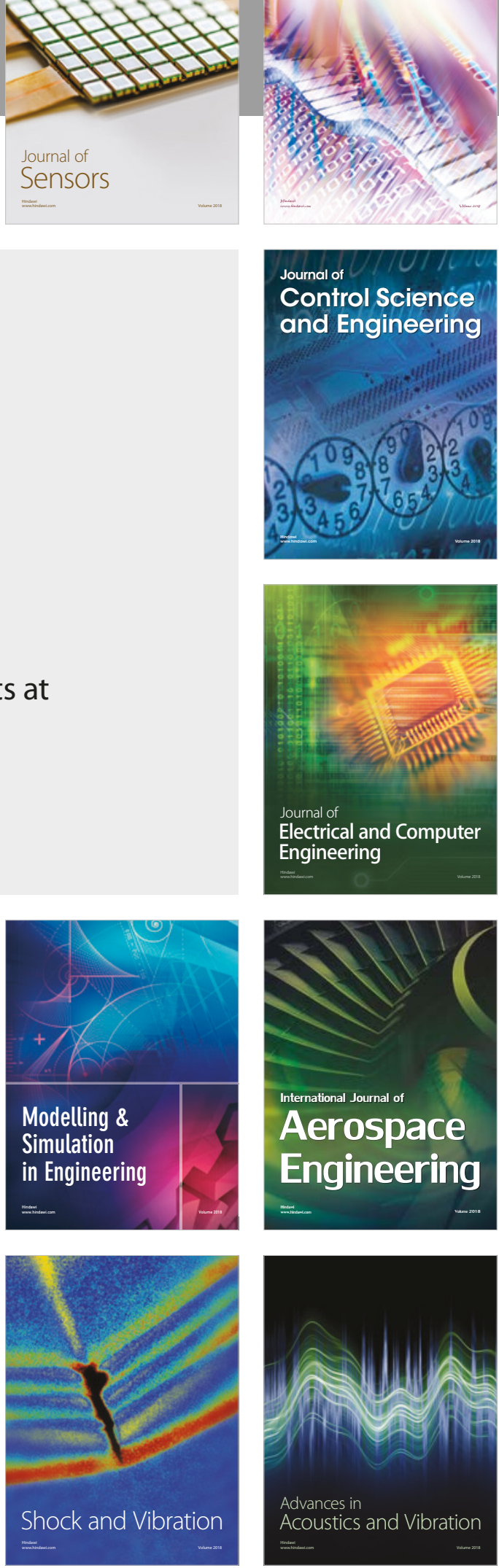